\title{
Some morphological characteristics of Praying Mantids (Insecta: Mantodea) of South Gujarat, India
}

\author{
H. N. Patel ${ }^{1 *}$ and Abhishek Shukla ${ }^{2}$ \\ ${ }^{1}$ Department of Agricultural Entomology, N.M.C.A, NAU, Navsari, India \\ ${ }^{2}$ Senior Acarologist AINP on Agricultural Acarology, Department of Agricultural \\ Entomology, N.M.C.A, NAU, Navsari, India \\ *Corresponding author
}

\begin{tabular}{|l|}
\hline Ke y w or d s \\
Mantodea, \\
mantids, \\
diversity, \\
Morphometrics
\end{tabular}

\section{Introduction}

Mantids are amongst the ambush pradators and most attractive creatures on earth due to its specialized front legs, in an attitude of prayer, camouflage and reproductive behavior(1-3). They are known to lay eggs in complex ootheca. Mantids play an imperative role as prey and predator to maintain the balance of tropic levels of food chain(4). These insects often remain motionless for hours, in search of prey, and only the head rotates about 180 degrees to watch any disturbances caused by flying insects. Globally 2400 species of mantids are known belonging to 142 genera and 18 families(5). A total 162 species exist in India belonging to 68 genera(6). Research on mantids in India was further propelled by several researchers in India (7- 12). 4 species 
and 4 genera of mantids have sofar been recorded from all over Gujarat (6). In the face of rapid decline of all organism and habitats owing to fast urbanisation and industrialisation in this Area, a list ofMantid fauna is extremely necessary to observe change in their biodiversity and morphological features. We are representing morphological characters of mantids (length of forewing, hind wing and abdomen) in South Gujarat, the present study was done. The main objectives of this study include to study the morphological characteristics of mantids in South Gujarat, India.

\section{Materials and Methods}

Study on morphology of Mantids in South Gujarat, India was carried out by the Department of Entomology, N.M. College of Agriculture, Navsari Agricultural University, Navsari, Gujarat during 2016-17, India. Photographs of species and their behavioural patterns were captured with the help of digital camera (Sony Alpha a7S gigital camera). Live specimens from the field were photographed, so that natural colouration and specific behavioural postures can be documented. The specimens of mantids were then processed for identification for which they were killed in killing jar and spread and pinned properly. The initial identification, of the praying mantids was done with the help of the keys of state fauna services of Zoological Survey of India, Kolkata.The final confirmation had been be done with the help of expert Dr.H.V.Ghate, Professor of Zoology, Modern College, Pune-411001, Maharashtra by sending specimens and personal visit on August 2nd, 2017. Who have 28 years of experience on order Mantodea. Simultaneously, collection of mantids were also done for measurement of their fore wing, hind wing and abdomen with the help of digital verniercalliper.

\section{Statistical analysis}

The experimental data was analysed statistically by using minimum, maximum values and average \pm standard deviation of the length of different body parts.

\section{Results and Discussion}

\section{Order: Mantodea}

\section{Family: Mantidea}

1. Mantis religiosa (Burmeister, 1838)

\section{Morphological description}

Colour: Yellowish green, brown, yellow, black;Head: triangular with a transverse dirty yellowish green patch across vertex and eye; Forelegs:coxal disc not flat, with six to seven submarginal granules, internally with marble callous spots; Middle and Hindlegs: slender, without lobulation, coxa broader and shorter than femur; Wings: both wings hyaline, costal area of forewing opaque; costal vein bifurcates distally; post radial vein bifurcates at middle, lower branch again bifurcates distally in both forewing and hindwing. This is one of the most common species of mantid found in almost all parts of world (Image-1).

Habitat: Paddy, Mango, Pond, Grassland and Banana ecosystem.

2. Hierodula keralenesis (Vyjayandi and Narendran, 1995)

\section{Morphological description}

Colour: Light green with slight ferrugeneousprozonal border; Head: triangular, Eyes: globular laterally, oval ventrally; Antenna: filiform, slender; Forelegs: stouter, coxa with many strong saw-like spines; Middle and Hind legs: slender, coxa 
shorter, femur and tibia almost equal in length; tibia more slender than femur; Forewing: with costal area moss green and opaque discoidal area light green, semihyaline, stigma yellow, veins light green (Image-2).

Habitat: Paddy and Grassland ecosystem.

\section{Hierodula coarctata(Saussure, 1869)}

\section{Morphological description}

Colour: Pale green, gray, black, yellow; Head: Thick, triangular wider ; vertex smooth, Eyes: globular laterally, ventrally subconical; Antenna: filiform, slender without setae; Pronotum: Elongate, supra coxaldialation oval, with indistinct mid longitudinal carina; Forelegs: superior margin of coxa with 4 to 5 obtuse thick spines, outer surface ridged, inferior end not serrated; Middle and Hind legs: coxa short; femur slightly shorter than tibia in mid leg, longer than tibia in hind leg; Wings: longer than abdomen, forewing with opaque, broad coastal area (Image-3).

Habitat: Paddy and Grassland ecosystem.

\section{Hierodula grandis(Saussure, 1869)}

\section{Morphological description}

This species is commonly known as giant Asian mantid and it is a large sized mantid which grows up to 6 inches long and is capable of eating small birds, reptiles and mammals; Colour: Green, brown; Head: Triangular, wider than high, vertex smooth, lateral lobes prominent; Eyes: globular laterally, subconical ventrally; ocelli large, closely placed; Antenna: slender, nonciliated; Pronotum: Oval shaped, slightly longer than fore coxa; Forelegs:coxa ridged dorsally; superior margin with five blunt spines; Middle and Hindlegs: Simple; Wings: Longer than abdomen, forewing costal area opaque (Image-4).
Habitat: Paddy, Mango, Pond and Grassland ecosystem.

\section{Hierodula viridis (Burmeister, 1838)}

Morphological description: Color: Pale green; Head: Thick, triangular, vertex smooth, lateral lobes slightly pronounced; Eyes: globular laterally, ocelli larger; Antenna: filiform, slender without setae; Forelegs: superior margin of coxa with four to five obtuse thick spines, outer surface ridged, inferior end not serrated. Middle and Hindlegs: coxa short, femur slightly shorter than tibia in midleg, longer than tibia in hindlegs; Wings: longer than metasoma, forewing with opaque, broad coastal area, hindwings hyaline (Image-5).

Habitat:Paddy, Mango, Pond, Grassland and Banana ecosystem.

\section{Hierodula venosa(Olivier, 1792)}

\section{Morphological description}

Colour: GoldenGreen; Head: Triangular, wider than high, vertex smooth, lateral lobes a little prominent; Eyes: globular dorsally, subconical ventrally, ocelli closely packed; Antenna: slender, without setae; Pronotum: Longer than forecoxa; supra coxaldialation oval, immediately narrows posterior to it; Forelegs : Coxa ridged externally, internally flat, with 6 to 7 strong, stout marginal spines; Middle and Hind legs: middle leg slightly shorter than hind legs, coxa short; Forewing: opaque except at apex, with dense reticulate veins; costal area broader (Image-6).

Habitat: Paddy, Mango, Pond, Grassland and Banana ecosystem.

7. Hierodula membranacea (Burmeister, 1838)

Morphological description: The bite of this 
large species can be painful and possibly break the skin; Colour: light green; Head: triangular; Antenna: slender, filiform; ocelli conspicuous; Forelegs: coxa inner margin tuberculated. Middle and Hind legs: slender; coxa short; hind femur longer than coxa, slightly shorter than tibia; metatarsus as long as all other tarsal segments together; Wings: longer than metasoma, forewings costal area opaque, posterior radial vein bifurcates proximally; hind wing hyaline, radial veins bifurcate (Image-7).

Habitat: Paddy, Mango, Pond, Grassland and Banana ecosystem.

\section{Ameles fasciipennis (Kaltenbach, 1963)}

\section{Morphological description}

Colour: green; Head: small; eyes prominent; ocelli conspicuous; Antenna: thick and bristled; Pronotum: short rhomboidal with black stripe extending upto head; metazona a little longer than prozona; fore femur dialated with 4 external and 4 discoidal spines; metatarsus longer than all other, tarsal segments together. Middle and Hind legs: much longer; Wings: wingless or sometime wings pad present (Image-8).

Habitat: Grassland ecosystem.

\section{Tenodora sinensis (Nurseryman, 1962)}

\section{Morphological description}

Colour:Black, straw yellow with greyish brown tinge; Head: Thick, triangular; Eyes: globular laterally, ventrally subconical, emarginate; ocelli larger; Antenna: filiform, slender without setae; Forelegs: superior margin of coxa with four to five obtuse thick spines, outer surface ridged, inferior end not serrated. Middle and Hind legs: elongated, delicate, coxa short; femur as long as tibia; metatarsus as long as all other tarsal segments together; Wings: sub hyaline, subconical at tips; forewing with reticulate venation, superior border opaque with transverse veins, without setae (Image-9).

Habitat: Paddy, Grassland and Banana ecosystem.

\section{Statiliamaculata(Thunberg, 1987)}

\section{Morphological description}

Colour:Green, fumy brown; Head: triangular; Antenna: slender, filiform; ocelli conspicuous; Forelegs: slender, coxa not flat with middorsaltuberculated ridge, ventrally submarginal area with 6 to 7 large well pronounced tubercles, internal apical lobes contiguous. Middle and Hind legs: coxa short; femur longer than coxa; Wings: both wings non-truncate with conical endings; costal area opaque, hindwings semihyaline, anterior radial vein bifurcates (Image-10).

Habitat: Paddy, Mango, Pond and Grassland ecosystem.

\section{Schizocephala bicornis (Linnaeus, 1758)}

\section{Morphological description}

Colour: Light green and Straw yellow with green tinge; Head: Triangular, narrow and long; Eyes: conical, pointed towards apex; Antenna: filiform, thick at base; Forelegs: elongate, coxa a little longer than femur, internal apical lobes divergent; Middle and Hindlegs: coxa short, femur as long as tibia in midlegs, tibia shorter than femur in hindlegs; Wings: short, leathery, reaches up to second metasomal segment, body length very long (Image-11).

Habitat: Paddy and Grassland ecosystem. 


\section{Archimantis latistyla (Serville, 1838)}

\section{Morphological description}

Colour: Green, brown Head: Triangular, wider than high, vertex smooth, eyes globular, ocelli closely placed; Antenna: slender, nonsetaceous; Pronotum: Elongated, longitudinally carinated; Middle and Hind legs: simple; Wings: both wings longer than abdomen forewings with opaque costal and semihyaline (Image-12).

Habitat: Paddy and Grassland ecosystem.

\section{Family: Hymenopodidae}

\section{Tropiodoguttatipennis (Stal, 1877)}

\section{Morphological description}

Colour: green; Head: dark wood brown with fuscous patches, Antenna: filiform; Forelegs: coxa dorsally with longitudinal ridge, ventrally smooth; Middle and Hindlegs: coxa short, middle leg shorter; Wings: forewings opaque, posterior radial vein bifurcates twice proximally; hindwings sub hyaline (Image13).

Habitat: Mango and Grassland ecosystem.

\section{Creobroter apialis (Audinet-Serville, 1839)}

\section{Morphological description}

Colour: green; Head: trapezoid, eyes conical, projecting upwards; Antenna: slender, filiform, ocelli conspicuous; Forelegs: coxa inner margin tuberculated. Middle and Hind legs: middle legs a little longer than hind legs, mid femur twice as long as mid tibia; Wings: longer than metasoma, costal and anal areas of forewing transparent; forewing green with yellow markings; hindwings coloured in female (Image-14).
Habitat: Paddy and Grassland ecosystem.

\section{Family: Liturgusidae}

15. Humbertiella ceylonica (Saussure, 1869)

\section{Morphological description}

Colour: Wood brown with black tinge; Head: dark wood brown with fuscous patches; Antenna: filiform; Forelegs: coxa dorsally with longitudinal ridge, ventrally smooth; Middle and Hindlegs: coxa short, middle leg shorter; Wings: forewings opaque, costal area reticulatelyveinated, posterior radial vein bifurcates twice proximally; hindwings sub hyaline (Image-15).

Habitat: Paddy and Grassland ecosystem.

\section{Family: Empusidae}

16. Empusa guttula(Thunberg, 1815)

\section{Morphological description}

Colour: light yellow, Deep brown, green; Head: Bluntly triangular, eyes round, projecting laterally; Antenna: Pectinate; Pronotum: Longer than fore coxa; depressed, Forelegs: simple, coxa : little shorter than femur, without spines; femur simple with a single median spine, tibia short, without external or internal spines, tibial claw well developed; Wings: longer than abdomen; forewing semi opaque, leathery (Image-16).

Habitat: Grassland ecosystem.

17. Gongylus gongylodes (Linnaeus, 1758)

\section{Morphological description}

This species known as the wandering violin mantis, ornate mantis or Indian rose mantis; 
Table1 Morphomatric variation of different mantids

\begin{tabular}{|c|c|c|c|c|c|c|c|c|c|c|}
\hline \multirow[t]{2}{*}{ S.No } & \multirow[t]{2}{*}{ Species of Mantids } & \multicolumn{3}{|c|}{ Abdomen (mm) } & \multicolumn{3}{|c|}{ Forewing(mm) } & \multicolumn{3}{|c|}{ Hindwing(mm) } \\
\hline & & Min. & Max. & Avg. \pm SD & Min. & Max. & Avg. \pm SD & Min. & Max. & Avg. \pm SD \\
\hline 1 & Mantis religiosa & 22.03 & 23.19 & $22.44 \pm 0.08$ & 19.98 & 20.14 & $20.07 \pm 0.06$ & 22.05 & 22.18 & $22.16 \pm 0.39$ \\
\hline 2 & Empusaguttula & 34.88 & 35.96 & $35.40 \pm 0.54$ & 34.96 & 36.04 & $35.57 \pm 0.55$ & 33.90 & 35.10 & $34.50 \pm 0.60$ \\
\hline 3 & Hierodulaviridis & 50.25 & 50.37 & $50.30 \pm 0.06$ & 46.25 & 46.39 & $46.32 \pm 0.07$ & 46.29 & 47.20 & $46.71 \pm 0.37$ \\
\hline 4 & Hierodulavenosa & 45.92 & 46.12 & $46.03 \pm 0.65$ & 48.03 & 48.38 & $48.16 \pm 0.18$ & 44.06 & 44.92 & $44.43 \pm 0.44$ \\
\hline 5 & Hierodulamembranacea & 44.10 & 45.88 & $44.98 \pm 0.69$ & 43.10 & 45.10 & $44.36 \pm 0.77$ & 40.12 & 42.20 & $41.38 \pm 0.90$ \\
\hline 6 & Hierodulacoarctata & 27.72 & 28.52 & $28.01 \pm 0.06$ & 29.52 & 30.64 & $30.12 \pm 0.41$ & 27.72 & 28.76 & $28.06 \pm 0.41$ \\
\hline 7 & Hierodulagrandis & 45.77 & 46.57 & $46.19 \pm 0.40$ & 47.83 & 48.92 & $48.39 \pm 0.54$ & 44.42 & 45.56 & $44.99 \pm 0.75$ \\
\hline 8 & Hierodulakeralenesis & 43.12 & 46.12 & $44.60 \pm 1.29$ & 41.10 & 43.10 & $42.10 \pm 0.86$ & 41.90 & 42.18 & $42.04 \pm 0.39$ \\
\hline 9 & Humbertiellaceylonica & 22.06 & 22.22 & $22.13 \pm 0.06$ & 25.98 & 26.81 & $26.39 \pm 0.40$ & 20.02 & 21.72 & $20.84 \pm 0.73$ \\
\hline 10 & Schizocephlabicornis & 81.91 & 82.88 & $82.50 \pm 0.52$ & 44.12 & 44.32 & $44.24 \pm 0.10$ & 42.12 & 42.37 & $42.25 \pm 0.12$ \\
\hline 11 & Gongylusgongylodes & 36.56 & 36.72 & $36.61 \pm 0.06$ & 26.62 & 26.78 & $26.69 \pm 0.07$ & 24.78 & 24.92 & $24.87 \pm 0.07$ \\
\hline 12 & Gongylustrachelophyllus & 33.08 & 33.30 & $33.19 \pm 0.15$ & 35.34 & 35.96 & $35.65 \pm 0.43$ & 34.46 & 34.72 & $34.59 \pm 0.72$ \\
\hline 13 & Creobroterapialis & 28.22 & 28.86 & $28.54 \pm 0.32$ & 30.08 & 30.22 & $30.15 \pm 0.07$ & 32.02 & 32.32 & $32.17 \pm 0.15$ \\
\hline 14 & Amelesfasciipennis & 14.46 & 14.68 & $14.57 \pm 0.16$ & - & - & - & - & - & - \\
\hline 15 & Tenodorasinensis & 39.98 & 40.12 & $40.05 \pm 0.05$ & 40.14 & 40.32 & $40.23 \pm 0.07$ & 44.62 & 44.77 & $44.70 \pm 0.05$ \\
\hline 16 & Statiliamaculata & 39.14 & 39.43 & $39.27 \pm 0.14$ & 37.98 & 38.46 & $38.18 \pm 0.24$ & 34.14 & 35.08 & $34.70 \pm 0.49$ \\
\hline 17 & Aethalochroaashmoliana & 50.40 & 51.20 & $51.00 \pm 0.43$ & 49.80 & 51.32 & $50.13 \pm 0.20$ & 50.12 & 51.02 & $50.60 \pm 0.36$ \\
\hline 18 & Aethalochroa insignis & 57.22 & 58.92 & $58.08 \pm 0.85$ & 43.08 & 44.11 & $43.45 \pm 0.56$ & 41.52 & 42.12 & $41.15 \pm 0.32$ \\
\hline 19 & Toxoderopsisspinigera & 79.98 & 81.03 & $80.65 \pm 0.58$ & 46.07 & 46.26 & $46.18 \pm 0.09$ & 39.81 & 40.72 & $40.18 \pm 0.47$ \\
\hline 20 & Archimantislatistyla & 45.92 & 48.12 & $47.02 \pm 1.55$ & 37.46 & 38.14 & $37.64 \pm 0.07$ & 35.05 & 36.18 & $35.61 \pm 0.39$ \\
\hline 21 & Tropiodoguttatipennis & 22.06 & 22.22 & $22.13 \pm 0.06$ & 19.98 & 20.14 & $20.07 \pm 0.07$ & 20.02 & 21.72 & $20.84 \pm 0.73$ \\
\hline
\end{tabular}


Photographs of different species of mantids (Mantodea)

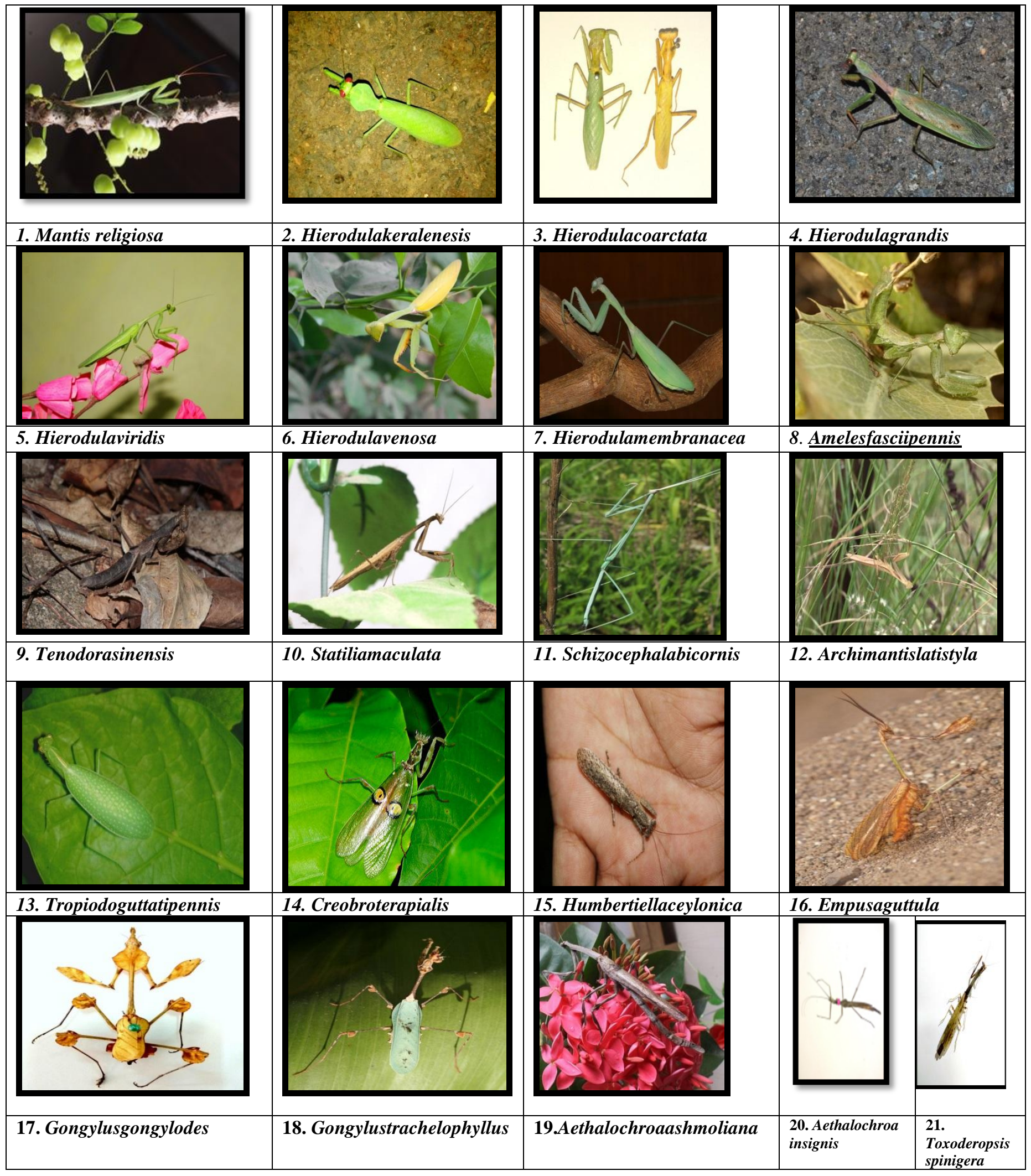


Colour: brown, yellow or yellowish green; Head: small, vertex with protuberance; Antenna: filiform in the case of female and pectinate in male; Fore legs and Hindlegs: slender, coxa with external lobes; femur with distal, triangular lobe dorsally and semicircular lobe ventrally; Wings: well developed; in males wings longer and in female shorter than metasoma (Image-17).

Habitat: Paddy and Grassland ecosystem.

\section{Gongylustra chelophyllus (Burmeister,} 1838)

\section{Morphological description}

Colour: brown, light green or yellowish green; Head: small, vertex with protuberance; antenna: filiform in the case of female, pectinate in male; Pronotum: slender with superior border of fore femur dialated; with 5 external and 4 discoidal spines; Mid and Hind legs: slender, coxa with external lobes; femur with distal, triangular lobe dorsally and semicircular lobe ventrally; Wings: well developed; in males rhomboidal dialation; fore coxa with backwardly directed, wings longer and in female shorter than abdomen (Image-18).

Habitat: Grassland ecosystem.

\section{V.Family: Toxoderidae}

19. Aethalochroa ashmoliana (Westwood, 1841)

\section{Morphological description}

Colour: brown; bizzare shaped; Head:small, wider than high; Antenna: filiform; Pronotum: spiny or tuberculated; prozonaspatulate, supra coxaldialation well pronounced; metazoancarinated, as long as fore coxa fore coxa with inner distal serrated lobe. Middle and Hind legs: short and stout; coxa short; femur slightly foliaceous with internal and external distal lobes, Forewings:semihyaline; costal area more opaque, hind wing longer than forewing (both wings shorter, up to three fourth of metasoma) (Image-19).

Habitat: Mango, Grassland and Banana ecosystem.

20. Aethalochroa insignis (Wood-Mason, 1878)

\section{Morphological description}

Colour:black, lightgrey, looks like dried leaves; Head: small, more or less globular; Eyes: globular, not projecting much; Antenna: simple, slender, filiform, Forelegs: simple, slender, coxa triangular in cross section; ridged middorsally, tuberculated inferiorly; Middle and hind legs: short and stout; coxa short; femur slightly foliaceous with internal and external distal lobes; Forewings: semihyaline; costal area more opaque, hind wing longer than forewing (both wings shorter, up to three fourth of metasoma) (Image-20).

Habitat: Mango, Pond, Grassland and Banana ecosystem.

The present findings are in agreement with consulting the literature Mantids fauna of Kerala published byVyjayandi with colourful photographs and Morphological features ofMantids. However, 21 species belonging 15 genera under five families were recorded from South Gujarat.

In conclusion, from the above mentioned results, it becomes clear that South Gujarat consisting huge diversity of mantids. Among 21 species of mantids Aethalochroa ashmoliana Westwood (Family: Toxoderidae) was the largest in relation to size of forewing $(50.13 \pm 0.20 \mathrm{~mm})$, hind wing $(50.60 \pm 0.36 \mathrm{~mm})$ and Schizocephala bicornis Linnaeus 
(Family: Mantidae) was the largest in relation to size of abdomen $(82.50 \pm 0.52 \mathrm{~mm})$, while Tripiodo guttatipennis Stal (Family: Hymenopodidae) was the smallest in size with measurement of forewing $(20.07 \pm 0.07 \mathrm{~mm})$, hind wing $(20.84 \pm 0.73 \mathrm{~mm})$ and abdomen $(22.13 \pm 0.06)$.

\section{Acknowledgement}

The authors are thankful to Dr. H. V. Ghate, Professor of Zoology, Modern College, Pune411001, Maharashtra, India for identification of Mantids, also thankful to Professor and Head, Department of Entomology, N. M. College of Agriculture, Navsari, The Principal and Dean, N. M. College of agriculture, Navsari and Director of Research and Dean P.G. Studies for providing all the necessary facilities during the course of study.

\section{References}

1. Sureshan PM, Sambath S. Mantid (Insecta: Mantodea) fauna of old Bihar (Bihar and Jharkhand) with some new records for the state. Records of the Zoological Survey of India. 2009; 109(3):11-26.

2. Sureshan PM. A Preliminary Study on the Mantid Fauna (Insecta: Mantodea) of Orissa, India. Rec. zool. Surv. India. 2009; 305:1-56.

3. Dutta W, Sur D. Praying Mantis: A threatened group of insect from Purulia, West Bengal. Biodiversity Conservation: Fundamentals and Applications. 2012; 262-263.

4. Sathe TV, Vaishali PJ. Report on nine new species of mantids (Insecta: Mantodea) and their insect pest predatory potential from agroecosystems of Kolhapur region, Journal of Entomology and Zoology Studies. 2014; 2(5):304-307.

5. Ehrmanm R. Mantodea: Gottesanbeterinnen der Welt. Naturund Tier-VeriagGombH (NTV), Munster, Germany.2002; 519.

6. Mukherjee TK, Hazra AK, Ghosh AK. The mantid fauna of India (Insecta: Mantodea). Oriental Insects.1995; 29:185-358.

7. Ghate HV, Ranade SP. Biodiversity of mantids (Insecta: Mantodea) in Pune (Western Ghats) with notes on other regions of Maharashtra, J Bombay Nat. Hist. Soc. 2002; 99(2):348-352.

8. Rao TK, Ghate HV, Sudhakar M, Maqsood JSM, Krishna SR. Updated checklist of praying mantid species (Insecta: Mantodea) from NagarjunasagarSrisailam Tiger Reserve, Andhra Pradesh. Zoos' Print Journal. 2005; 20(6):1905-1907.

9. Sureshan PM, Jafer P, Radhakrishnan C. New additions to the mantid fauna (Insecta: Mantodea) of Andaman \& Nicobar Islands, India. Zoos' Print Journal. 2004; 19(7):1544.

10. Sureshan PM, Ghate HV, Radhakrishnan C. Insecta: Mantodea. Fauna of TadobaAndhari tiger Reserve. Zoo1. Surv. India. Conservation Area Series.2006; 25:227-232.

11. Vyjayandi MC, Narendran TC, Mukherjee TK. A new species of praying mantis (Insecta: Mantodea) from Kerala, India. Oriental Insects.2006; 40:285-290.

12. Vyjayandi MC. Mantid fauna of Kerala, India. Rec. zoo1.Surv. India. Occ. 2007; 267:1169.

\section{How to cite this article:}

Patel, H. N., and Abhishek Shukla. 2018. Some morphological characteristics of Praying Mantids (Insecta: Mantodea) of South Gujarat, India. Int.J.Curr.Microbiol.App.Sci. 7(06): 3612-3620. doi: https://doi.org/10.20546/ijcmas.2018.706.425 